\title{
Fatal Outcome Due to Sepsis by Mycobacterium Bovis Six Years After BCG Intravesical Instillations
}

\author{
Miguel Alvarez-Mugica ${ }^{\mathrm{a}, \mathrm{e}}$, Jesus Ma Fernandez Gomez ${ }^{\mathrm{b}}$, Veronica Bulnes Vazquez ${ }^{\mathrm{c}}$, Antonio Jalon Mon- \\ zon $^{\mathrm{b}}$, Jorge Garcia Rodriguez ${ }^{\mathrm{b}}$, Jose Ma Fernandez Rodriguez ${ }^{\mathrm{d}}$, Victor Asensi ${ }^{\mathrm{d}}$, Jose Antonio Carton ${ }^{\mathrm{d}}$
}

\begin{abstract}
Bacillus Calmette-Guerin (BCG) in intravesical instillations is the reference treatment for urothelial carcinoma with a high risk of progression. Morbidity secondary to intravesical BCG may present both locally and systemically. Most patients suffer a self-limited irritative voiding syndrome. Prevention of these complications requires implementing rules of good practice for the instillations. The undesirable side effects should be recognized early. Their treatment should be rapid and adapted to the patient. Besides the commonly seen side effects of intravesical BCG instillations, very rare complications have been reported. In some patients, infection appears early (within 3 months after instillation) and is characterized by generalized symptoms, with pneumonitis and hepatitis. Late-presentation disease occurs more than 1 year after the first BCG treatment and usually involves focal infection of the genitourinary tract (the site at which bacteria were introduced) and/or other sites that are typical for reactivation of mycobacterial disease, such as the vertebral spine or the retroperitoneal tissues. Non caseating granulomas are found in the majority of cases, whether early or late. Most patients respond to treatment with antituberculous drugs; in early-presentation disease, when features of hypersensitivity predominate, glucocorticosteroids are sometimes added. Late localized infection often requires surgical resection.
\end{abstract}

Keywords: Bacillus Calmette-Guerin; Instillations; Transitional cell carcinoma; Septicaemia

\footnotetext{
Manuscript accepted for publication September 9, 2010

${ }^{a}$ Department of Urology, Hospital Valle del Nalon, Langreo, Asturias, Spain

${ }^{\mathrm{b}}$ Department of Radiology, Hospital Alvarez Buylla, Mieres, Asturias, Spain

${ }^{c}$ Department of Urology, Hospital Universitario Central de Asturias, Oviedo, Asturias, Spain

${ }^{\mathrm{d}}$ Department of Internal Medicine, Hospital Universitario Central de Asturias, Oviedo, Asturias, Spain

${ }^{e}$ Corresponding author: Urology department, Valle del Nalon Hospital, Poligono de Riano s/n, Langreo, Asturias, Spain

Email: malvarezmug@gmail.com
}

\section{Introduction}

Bacillus Calmette-Guerin (BCG) is a live attenuated strain of Mycobacterium bovis that has been used to treat transitional-cell carcinoma since 1976 [1] and has been reported to eradicate disease in more than $70 \%$ of patients with in situ and stage I disease [2]. Intravesical therapy with BCG is generally considered safe, however, serious complications including hematuria, granulomatous pneumonitis, suppurative lymphadenitis, distant intramuscular and bone abscesses, hepatitis, and life-threatening BCG sepsis are well known [3], although late bacteriuria and sepsis due to BCG instillations, have not been described yet. The reported incidence of other than minor complications is under 5\% [4]. Recommended treatment for disseminated BCG disease includes a combination of antituberculous medications (with the exception of pyrazinamide, to which BCG is typically resistant) and a tapering course of steroids [5].

These complications are an absolute contraindication for further BCG instillations. Despite its toxicity, the risk-benefit ratio favors the use of BCG in patients who have moderate and high risk tumors [2]. There are not differences of toxicity between Connaught and Pasteur strain in intravesical BCGtherapy of superficial bladder tumors [6].

Compliance with this treatment is altered by its potentially serious locoregional or general side effects. Prevention of these complications requires implementing rules of good practice for the instillations. The undesirable side effects should be recognized early. Their treatment should be rapid and adapted to the patient. In order to prevent complications from BCG immunotherapy, a French study showed that side effects were significantly reduced by administration of ofloxacin after each instillation of BCG. The number of side effects requiring antitubercular treatment was also reduced in the patients in this study who had received ofloxacin [7]. Puigvert Foundation in Spain developed a practical guideline for the management of the complications followed BCG instillations [8].

Although fatal sepsis has been described before [9-11], in a search of PubMed we did not find any fatal sepsis as late complication of BCG instillations. What we report, to our

doi:10.4021/jmc42w 
knowledge, is the first case of disseminated BCG infection causing septicemia and death 6 years after intravesical treatment with BCG therapy for bladder cancer.

\section{Case Report}

An 83-year-old man was hospitalized with a 2-week history of fever, malaise, anorexia and bleeding from a back drainage. The patient had previously undergone two trans-urethral resections receiving 12 intravesical bacillus Calmette-Guerin instillations without any complications for a high grade transitional cell carcinoma of the bladder. Due to tumor progression he was performed a radical cystoprostatectomy 5 years earlier of this admission. He also had an endovascular stentgraft repair of an infrarenal abdominal aortic aneurysm. Six months before this new admission, he was diagnosed from multiple retroperitoneal abscesses in which mycobacterium bovis grew. Since then, the patient received a combination of antitubercular medication.

Physical examination revealed an ill-appearing man. Pulmonary and cardiac examinations were unremarkable. He complained of abdominal pain in the right flank. Laboratory tests revealed abnormal liver function tests, anemia, renal insufficiency, leukocytosis and signs of coagulation disorder. A computed tomography (CT) scan of the abdomen showed a diffused and enlarged left psoas muscle. High doses of corticosteroids were associated to the antitubercular treatment. The patient developed a severe sepsis. Blood cultures were positive for acid-fast bacilli stains. Despite partial improvement, the course was complicated with a multiple organ failure, and the patient died two weeks after admission.

\section{Discussion}

Permanent increase in neoplasm incidence including bladder neoplasm makes physicians to search for new forms and methods of treatment that prevents either appearance and/or progression of the disease. Application of new preparations entails in many cases appearance of side effects which sometimes are difficult to manage and thus must be monitored constantly. To avoid complications which in case of BCG application are very burdensome and sometimes dangerous for patient, it is necessary to intervene in due time.

The BCG therapy has been effectively used in the management of superficial bladder cancers. It is especially useful as an adjuvant therapy following bladder surgery for cancer. For instance, Demkow et al [2], in a recent study, reported that nearly $66 \%$ of the patients who received intravesical BCG therapy following complete transurethral resection of a bladder tumor were cancer free after a median follow-up of 45 months.

Adverse events following intravesical BCG therapy are related to strain virulence, allergic reactions or to nosocomial urinary tract infections. BCG is a potent immunostimulator that exerts its urological effects by inducing a strong immune response and by causing cell cycle arrest at the G1/S transition phase $[12,13]$. A multicenter reviewed and studied complications on 1,278 patients after bacillus CalmetteGuerin therapy for bladder cancer [14]. Cystitis occurred in $91 \%$ of the patients. Complications identified included fever in 50 patients $(3.9 \%)$, granulomatous prostatitis in 17 $(1.3 \%)$, bacillus Calmette-Guerin pneumonitis or hepatitis in $12(0.9 \%)$, arthritis or arthralgia in $6(0.5 \%)$, hematuria requiring catheterization or transfusion in $6(0.5 \%)$, skin rash in $5(0.4 \%)$, skin abscess in $5(0.4 \%)$, ureteral obstruction in $4(0.3 \%)$, epididymo-orchitis in $2(0.2 \%)$, bladder contracture in $2(0.2 \%)$, hypotension in $1(0.1 \%)$, and cytopenia in $1(0.1 \%)$. Deaths due to BCG sepsis and the high frequency of BCG-induced cystitis have compromised the use of BCG. However, with increased experience in applying BCG, the side-effects now appear to be less prominent. Serious sideeffects are encountered in fewer than 5\% [15]. Major complications can appear after systemic absorption of the drug, therefore BCG should not be administered during the first 2 weeks after transurethral resection, in patients with hematuria and after traumatic catheterization. Morbidity secondary to intravesical BCG may present both locally and systemically. Most patients suffer a self-limited irritative voiding syndrome. Prevention of these complications requires implementing rules of good practice for the instillations. The undesirable side effects should be recognized early. Their treatment should be rapid and adapted to the patient. Besides the commonly seen side effects of intravesical BCG instillations, very rare complications have been reported.

\section{References}

1. Morales A, Eidinger D, Bruce AW. Intracavitary Bacillus Calmette-Guerin in the treatment of superficial bladder tumors. J Urol 1976;116(2):180-183.

2. Demkow T, Alter A, Wiechno P. Intravesical bacillus Calmette-Guerin therapy for $\mathrm{T} 1$ superficial bladder cancer. Urol Int 2008;80(1):74-79.

3. Rawls WH, Lamm DL, Lowe BA, Crawford ED, Sarosdy MF, Montie JE, Grossman HB, et al. Fatal sepsis following intravesical bacillus Calmette-Guerin administration for bladder cancer. J Urol 1990;144(6):13281330 .

4. Gonzalez OY, Musher DM, Brar I, Furgeson S, Boktour MR, Septimus EJ, Hamill RJ, et al. Spectrum of bacille Calmette-Guerin (BCG) infection after intravesical BCG immunotherapy. Clin Infect Dis 2003;36(2):140148.

5. Rischmann P, Desgrandchamps F, Malavaud B, Chopin DK. BCG intravesical instillations: recommendations 
for side-effects management. Eur Urol 2000;37 Suppl 1(33-36.

6. Champetier D, Valignat C, Lopez JG, Ruffion A, Devonec M, Perrin P. [Intravesical BCG-therapy: comparison of side effects of Connaught (Toronto) and Pasteur (Paris) strains]. Prog Urol 2000;10(4):542-547.

7. Colombel M, Picard A. [Prevention of Bacillus Calmette-Guerin immunotherapy complications]. Prog Urol 2008;18 Suppl 5(S105-110.

8. Rodriguez F, Palou J, Martinez R, Rodriguez O, Rosales A, Huguet J, Villavicencio H. [Practical guideline for the management of adverse events associated with BCG installations]. Arch Esp Urol 2008;61(5):591-596.

9. Nadasy KA, Patel RS, Emmett M, Murillo RA, Tribble MA, Black RD, Sutker WL. Four cases of disseminated Mycobacterium bovis infection following intravesical BCG instillation for treatment of bladder carcinoma. South Med J 2008;101(1):91-95.

10. Borre S, Brustia D, Rosa F, Brondolo R, Rizzo G, Garavelli PL. [Calmette-Guerin bacillus disseminated infection after intravesical instillation]. Recenti Prog Med 2002;93(4):247-248.
11. Elmer A, Bermes U, Drath L, Buscher E, Viertel A. [Sepsis and multiple organ failure after BCG-instillation for bladder cancer]. Internist (Berl) 2004;45(8):935-939.

12. Chen F, Zhang G, Cao Y, Payne R, See WA. Bacillus Calmette-Guerin inhibits apoptosis in human urothelial carcinoma cell lines in response to cytotoxic injury. J Urol 2007;178(5):2166-2170.

13. Shintani Y, Sawada Y, Inagaki T, Kohjimoto Y, Uekado Y, Shinka T. Intravesical instillation therapy with bacillus Calmette-Guerin for superficial bladder cancer: study of the mechanism of bacillus Calmette-Guerin immunotherapy. Int J Urol 2007;14(2):140-146.

14. Lamm DL, Stogdill VD, Stogdill BJ, Crispen RG. Complications of bacillus Calmette-Guerin immunotherapy in 1,278 patients with bladder cancer. J Urol 1986;135(2):272-274.

15. van der Meijden AP, Sylvester RJ, Oosterlinck W, Hoeltl W, Bono AV. Maintenance Bacillus Calmette-Guerin for Ta T1 bladder tumors is not associated with increased toxicity: results from a European Organisation for Research and Treatment of Cancer Genito-Urinary Group Phase III Trial. Eur Urol 2003;44(4):429-434. 